\title{
La Gestion du Risque de Micro-crédit: La Nécessité du Diagnostic Financier: Cas de la PME MIGEG Microfinance S.A.
}

\author{
Lucas Pony \\ Enseignant/Expert en Banque et Microfinance, \\ Université de Dschang, Cameroun
}

doi: 10.19044/esj.2017.v13n19p292 URL:http://dx.doi.org/10.19044/esj.2017.v13n19p292

\begin{abstract}
The reduction of the losses related to credit outstanding payment is very necessary in the micro finance institutions in general, and the SME of micro finance MIGEG* S.A in particular. Here, we carried out a study concerning the management of micro credits risk which result to losses due to non-refund. The method that was proposed in this study is that of the financial diagnosis which operates in two phases. The first phase involves the use of the data collected during the interview to analyze the morality of the customer (Phase 1), while the second phase involves the use of the financial and accounting quantitative data to analyze the financial health of the customer (Phase 2).The diagnosis method considered is that of Dupont which allows us to considerably reduce the percentage of credits outstanding payment. Consequently, it also helps to improve the company's funds. The proposed method will help the managers to detect the risks of application for credit. The bank will thus grant only the credit bonds related to the highest refunds. This, however, will further lead to the following results: For MIGEG S.A to be viable, it must insist on the ratio of the assets. This refers to the sales of the credits to promote refunds without risk. On the other hand, MIGEG S.A will insist on the ratio of the financial lever, debt and assets, in order to promote the prefinancing of the risk credits. Therefore, it will help to avoid credits related to the guarantees such as the land certificate, inheritance etc.
\end{abstract}

Keywords: management, risk, microcredit, diagnosis, SME, microfinance.

\section{Résumé}

La réduction des pertes liées aux impayés est urgemment nécessaire dans les établissements de micro finance, particulièrement la PME de micro finance MIGEG* S.A où nous avons effectué une étude concernant la 
gestion du risque de micro crédits qui cause des pertes en cas de non remboursement. La méthode que nous proposons est celle du diagnostic financier qui se déroule en deux phases à savoir : l'utilisation des données collectées lors de l’interview, afin d’analyser la moralité du client (phase1), l'utilisation des données quantitatives financières et comptables pour analyser la santé financière du client (phase 2). La modalité de diagnostic choisie est celle de Dupont qui permet de réduire considérablement le pourcentage des impayés, ce qui entraine l'amélioration de la trésorerie de l'entreprise. Cette méthode va permettre aux gestionnaires de déceler les risques de demande de crédit. Ainsi la banque ne devra octroyer que les bons crédits liés aux remboursements élevés. Ce qui conduit ainsi aux résultats selon lesquels, la MIGEG S.A pour être viable doit insister sur le ratio des actifs: les ventes sur les actifs pour favoriser les remboursements sans risque. Aussi, la MIGEG S.A doit insister sur le ratio du levier financier : dette plus avoir afin de favoriser le préfinancement des crédits à risque. Ce qui conduit ainsi à l'abandon des crédits liés aux garanties comme le titre fonciers, le patrimoine etc.

Mots-clés: gestion, risque, micro-crédit, diagnostic, PME, micro finance

\section{INTRODUCTION GENERALE}

\section{A- Contexte de l'étude}

La micro finance est apparue en Asie au milieu des années 1970 et dans une certaine mesure en Amérique latine.

L’exemple le plus cité est celui de Grameen Bank fondée en 1978 par Mohammed Yunus qui cherchait une réponse concrète à la crise de la famine qui traversait son pays le Bangladesh. Il trouva à cet effet, un groupe de 42 femmes qui fabriquaient des chaises en bambou et leur proposa des fonds propres pour acheter la matière première afin qu'elles puissent être autonomes.

Il leur prêta ensuite, une somme évaluée à environ 27 dollars sans intérêt permettant au groupe de femmes, de vendre leurs tabourets à des meilleurs prix et de sortir ainsi du cycle d'endettement dans lequel elles étaient enfoncées et qui les liaient aux marchands locaux. C'est ainsi que la Grameen Bank ou banque du village est née au Bangladesh et a favorisé le développement du microcrédit.

Toutefois au Cameroun, la micro finance a existé depuis plus d'un siècle sous sa forme traditionnelle avec les tontines. Elle a ainsi démarré sous sa forme formelle en 1963 avec la création de la première coopérative d'épargne et de crédit «Credit union » sous l'impulsion des missionnaires hollandais (Bouyop et al., 2012). 
Au cours des années 1980, l'environnement bancaire national est menacé du fait de l'absence d'un régulateur et du manque de professionnalisme des agents, ce qui va entrainer le durcissement des conditions d'accès au crédit du fait des montants élevés des impayés et des crédits non remboursables. La crise du secteur bancaire va donc entrainer la liquidation de plusieurs banques. La fermeture de nombreux guichets et le licenciement de nombreux cadres. Ces derniers n'ayant plus d'emplois, n'auront pour seule formation ou expérience que la finance et la reconversion.

C’est ainsi qu'ont été créé de nombreuses coopératives d'épargne et de crédit se rapprochant des banques (Bouyop et al., 2012). C'est par la suite à travers le décret n98/300/ PM du 9/9/1998 et le décret n²001/023/ PM du 29/07/2001 qu'a été institué respectivement le IMF (institution de micro finance (statut national)) et l'EMF (établissement de microfinance (statut régional))

B- La problématique

Le terme microfinance est utilisé pour désigner l'ensemble des services financiers offerts aux populations travaillant dans différents secteurs socioéconomiques qui gèrent des petites et des micros entreprises (Mohammed Yunus, 1997)

Parmi ces services nous pouvons citer entre autre, la mobilisation des dépôts (épargne), l’octroi des crédits (productifs et de micro assurance), les services d'assurance, les services de transfert de fonds (Bouyop et al., 2012).

Ces produits sont pour la plupart principalement destinés aux entrepreneurs, communautés et des personnes marginalisées ne bénéficiant pas de ces services (Bate, 2006).

Le crédit bancaire devient donc de ce fait une relation d'urgence par laquelle le prêteur (le principal) "loue » une part de richesse aux microentrepreneurs (les agents) qui s'engagent à rembourser le principal et à lui payer les charges d'intérêt à l'échéance et aux conditions fixées dans un contrat établi au préalable par les parties. Un problème d'urgence se pose car les intérêts de l'emprunteur et du prêteur différent alors que l'un (l'agent) est essentiellement concerné par la rentabilité des capitaux empruntés, l'autre l'est par la solvabilité du premier (Julien \& Pallaque, 1995) et la rentabilité des fonds propres.

Le principal objectif de la microfinance est de donner aux personnes considérées comme pauvres l'accès aux services financiers (épargne et crédit) afin de limiter les contraintes financières auxquelles ils font face et de lutter contre la pauvreté.

Cependant, toute microfinance cherche à obtenir le taux de remboursement le plus élevé possibles. Ces taux de remboursement élevés 
sont en effet associés à des bénéfices élevés pour une microfinance pour ses emprunteurs.

Afin de perdurer et de maintenir son activité dans le temps, la microfinance comme la MIGEG doit mieux gérer les risques auxquels il est soumis, en particulier celui du remboursement des crédits du fait de l’insolvabilité ou de la mauvaise foi des clients.

Etant donné que l'une des finalités d’un crédit c’est son remboursement, selon (Alioune Sall, 2000), « Bien que la structure des actifs des banques africaines reflètent en partie le fait que la masse des engagements remboursable à vue, leur préférence pour l'octroi des prêts essentiellement à court terme peut également s'expliquer par une attitude d'aversion contre les risques dans une situation bien précise ».

Quant à (Antoine Gentier, 2003), « une politique de crédit considérée comme stable lorsque le profil de crédit en longue période n’est pas affecté par des variations qui peuvent être suivies par des contractions très brutales ».

Et d’après (F. Jacques,1983), «pour limiter le risque de crédit bancaire, les banques peuvent répartir leurs concours entre des branches d'activités différente et au bénéficiaire uniquement ».

Ce qui amène à se poser la question : Quels sont les effets de l'analyse préalable du crédit sur le remboursement effectif du crédit? Autrement dit, quels sont les méthodes de recouvrement utilisées par la MIGEG S.A. dans le cadre du recouvrement de ses crédits à risque?

C'est ce qui va nous conduire aux objectifs ci-après:

C- Objectif général

La maîtrise du risque de crédit par les avoirs des actionnaires devant servir de préfinancement du risque.

D- Objectif spécifique

Contribuer à l'amélioration des méthodes utilisées contre les risques par la proposition des méthodes plus adéquates et durables.

E- Hypothèse

La valeur prise par le ratio de marge nette est suffisamment élevée pour favoriser le préfinancement des crédits à risque.

F- Méthode

La méthode à adopter ici sera principalement celle du modèle de Dupont qui part d'une équation standard et se décompose en trois premiers ratios et leur simplification. Ce qui expliquera le rendement à la MIGEG permettant de supporter les crédits à risque. Notamment dans le contexte de notre modèle qui met en relation l'ouverture extérieur, l'emploi, le capital humain, l’inflation, l’investissement intérieur puisque le préfinancement du 
risque et les ventes élevée vont créer les emplois par l'accroissement des investissements.

G- Recommandation

Trois composantes principales fondent l'analyse d'après ce modèle de Dupont : la rentabilité des ventes, l'efficacité de la gestion des actifs et l'importance relative de l'endettement. La performance de l'entreprise relative à chacune des composantes insiste en définitive sur le ratio de rotation des actifs car les ventes doivent être très élevées pour assurer une trésorerie viable. Le ratio du levier financier dette plus avoir doit également être très élevé pour assurer le préfinancement du risque. Le gestionnaire de crédit doit suivre tous les gestes et actions de l'emprunteur afin de réduire au mieux le risque de crédit.

H- Plan à suivre

On partira des composants du rendement des avoirs en entreprise $\left(1^{\text {ère }}\right.$ partie) pour dégager les conséquences sur le crédit à risque en entreprise (2eme partie).

\section{I-LE CREDIT A RISQUE EN ENTREPRISE}

A- Essai d'analyse

Le risque dépend de la probabilité objective qu'une institution a de réaliser les pertes (Lanha, 2006). Certains risques peuvent avoir des effets positifs. On parle alors d'opportunités. D’autres entrainent des effets négatifs dont l'entreprise veut s'en débarrasser. En ce qui concerne les établissements de crédit, ils sont soumis à plusieurs types de risque dont d'autres sont directement générés par l'activité de l'entreprise à savoir les risques endogènes et d'autres risques qui naissent dans l'environnement de l'entreprise et sont les risques exogènes.

Parmi les nouvelles méthodes de gestion de risque bancaire développées, nous avons la méthode value at risk (VAR). Cette méthode est considérée aujourd'hui comme une référence en matière de gestion des risques.

La VAR est la perte maximale sur un crédit (Vernimmen, 2011). Dans sa forme générale, elle est tirée de la distribution de probabilité des pertes et est égale aux quantités d'ordre de la fonction de densité des pertes. Les éléments nécessaires pour le calcul de la VAR sont la moyenne et l'écart type des prix des actifs. Si ces éléments sont facilement estimables pour les valeurs faisant l'objet d'une cotation, leur estimation dans le cas des crédits bancaires présente une difficulté car leur valeur n’est pas directement observable.

Ce qui va nous conduire à présenter la méthodologie nécessaire pour conduire l'approche économétrique. Nous partirons de la théorie 
économique néoclassique qui met en relation la production, le capital et l'emploi pour élaborer notre modèle économétrique. Ainsi, nous avons jugé adéquat d'utiliser la modélisation VAR pour analyser l'interrelation entre les variables. Ainsi, il s'agit pour nous de présenter les différentes sections qui nous permettrons de faire une brève description de notre modèle. A cet effet, nous indiquerons les sources de nos données, présenterons nos variables, spécifierons notre modèle économétrique, enfin nous présenterons les différents tests statistiques et de causalités.

Nous avons mobilisé à la suite de notre revue de la littérature les données de source secondaire pour la présente étude. Ces données proviennent de la Banque Mondiale, plus précisément dans le « Word data indicator $»$.

Nous avons fait une étude en coupe longitudinale sur la période allant de 1979 à 2014. Pour mener à bien notre étude, nous avons utilisé commutativement les logiciels tels que Word et Excel pour s'assurer du traitement de nos données et Eviews pour l'analyse de nos données.

B- Modèle économétrique abréviation des variables

Avant toute construction méthodologique, nous croyons judicieux de présenter tout d'abord dans un tableau la synthèse des différentes variables retenues dans le cadre de notre étude.

\section{1- Abréviation des variables}

\section{Abréviations Variables}

LOUV $_{\mathrm{t}}$

Logarithme de l'ouverture extérieure mesuré par les flux d'exportations et d'importations de biens et services sur PIB

$\mathrm{LKH}_{\mathrm{t}} \quad$ Logarithme du capital humain mesuré par la population scolarisée du niveau d'éducation secondaire

LEMP $_{t} \quad$ Logarithme de l'emploi mesuré par le volume d'emploi

LINF $_{\mathrm{t}} \quad$ Logarithme du taux d'inflation

LTIIB $_{\mathrm{t}} \quad$ Logarithme de volume des investissements intérieurs bruts

\section{2-Modèle à estimer}

Notre modèle se base sur la théorie économique néoclassique de Solow-Swan (1950) qui établit une relation entre la production (Y), l'emploi (L) et le capital (K).

$$
\mathrm{Y}=\mathrm{f}(\mathrm{K}, \mathrm{L})
$$

Le capital humain $(\mathrm{KH})$ est inclus dans cette fonction car il peut être un alternatif à l'amélioration technologique et peut induire la croissance et l'emploi en l'absence des progrès techniques (Lucas, 1988). Introduire 
l'investissement (TIIB) est aussi important car il est déterminant pour la relance de la production, de l'éducation, accroit la qualification du capital humain et par conséquent du niveau de l'emploi (Barro \& Sala-i-Martin, 1995). En considérant l'idée selon laquelle aucun pays à l'ère de la mondialisation ne saurait vivre en autarcie, nous introduirons dans notre modèle la variable ouverture extérieure (OUV). Ce qui suppose ici que le ratio de rotation des actifs : ventes /actifs est trop élevé pour assurer une trésorerie de l'entreprise viable pour supporter l'ouverture extérieure.

De plus, le ratio de levier financier : Dette + avoir doit également être très élevé pour contribuer au préfinancement du risque d'ouverture extérieure

Williams Phillips (1958) montre à travers sa fameuse courbe de Phillips qu'il existe une corrélation négative entre l'inflation et le chômage, nous incitant à cet effet à introduire dans notre modèle le taux d'inflation (INF). L'emploi dans ce modèle de base sera maintenu et sera mesuré par le volume d'emploi (EMP). Ce qui justifie ainsi le fait que le ratio de rotation des actifs et le ratio du levier financier sont très important dans la prise en compte de l'ouverture extérieure dans le modèle.

Ainsi donc, en partant de l'équation formulée plus haut, nous allons spécifier une modélisation quant à notre étude. Puisque notre objectif vise à analyser la relation entre l'ouverture et le capital humain, tout en établissant le lien avec l'emploi, nous remplacerons la variable Y par l'emploi (EMP). K sera pris comme le capital humain $(\mathrm{KH})$. Dès lors, le modèle économétrique à estimer sera donc sous la forme:

$\mathrm{EMP}=\mathrm{f}(\mathrm{KH}, \mathrm{OUV}, \mathrm{INF}, \mathrm{TIIB})$

Toutefois, nous récrirons notre modèle sous la forme logarithmique. Car, l'un des avantages de l'utilisation de la forme logarithmique est qu'elle permet de :

- minimiser l'influence des effets du temps sur la série ;

- réduire le nombre d'étapes pour aboutir à une série stationnaire ;

- interpréter les coefficients en élasticité ce qui nous facilite l'analyse économique à savoir l'impact d'un taux de variation de la variable explicative de $1 \%$ sur le taux de variation de la variable expliquée donnée.

Ainsi, nous avons en définitive : LEMP $=\mathrm{f}(\mathrm{LKH}, \mathrm{LOUV}$, LINF, LTIIB).

Afin de mieux cerner le lien causal existant entre les différentes variables, la modélisation VAR est la mieux indiquée. Car, elle présente nombreux avantages : outre le fait qu'elle résout le problème d'endogénéité potentielle des variables inhérentes aux modèles structurels, la modélisation VAR permet aussi d'autres applications différentes telles que des prévisions, 
des analyses en termes de causalité, des simulations des chocs structurels. Par rapport à d'autres modélisations macro économétriques, le VAR présente l’intérêt d’être plus simple dans sa représentation.

Équation 1: La spécification VAR du modèle.

$\left\{\begin{array}{c}\text { LEMP }_{t}=\mathrm{a}_{0}+\mathrm{a}_{1} \text { LEMP }_{\mathrm{t}-\mathrm{i}}+\mathrm{a}_{2} \text { LKH }_{\mathrm{t}}+\mathrm{a}_{3} \text { LOUV }_{\mathrm{t}}+\mathrm{a}_{4} \text { LTIIB }_{\mathrm{t}}+\varepsilon_{1 \mathrm{t}} \\ \text { LKH }_{\mathrm{t}}=\mathrm{b}_{0}+\mathrm{b}_{1} \text { LKH }_{\mathrm{t}-\mathrm{i}}+\mathrm{b}_{2} \text { LOUV }_{\mathrm{t}}+\mathrm{b}_{3} \text { LEMP }_{\mathrm{t}}+\mathrm{b}_{4} L \text { LIIB }_{\mathrm{t}}+\varepsilon_{2 \mathrm{t}} \\ \text { LOUV }_{\mathrm{t}}=\mathrm{c}_{0}+\mathrm{c}_{1} \text { LOUV }_{\mathrm{t}-\mathrm{i}}+\mathrm{c}_{2} \text { LKH }_{\mathrm{t}}+\mathrm{c}_{3} \text { LEMP }_{\mathrm{t}}+\mathrm{c}_{4} \text { LINF }_{\mathrm{t}}+\mathrm{c}_{5} L \mathrm{TIIB}_{\mathrm{t}}+\varepsilon_{1 \mathrm{t}}\end{array}\right.$

Ici, i est le nombre de retard

Nous effectuerons une investigation empirique en série temporelle ${ }^{3}$.

\section{C- Présentation des variables}

Comme toute modélisation, le VAR comporte deux types de variables : les variables endogènes et les variables exogènes ou les variables expliquées ou explicatives. En principe, chaque variable endogène ou expliquée est encore potentiellement exogène ou explicative. Nous distinguons aussi parmi nos variables, des variables explicatives retardées de t-i période.

\section{i. Variables endogènes}

Les séries temporelles peuvent être définies comme un ensemble d'observations ordonnées dans le temps. En ajoutant une dimension temporelle il est possible d'expliquer les variables endogènes non seulement par les variables explicatives présentes mais également par des variables explicatives passées, en particulier par des valeurs passées de la variable endogène. Ils peuvent être utilisés pour résoudre deux types de problèmes : celui de l'auto-corrélation des résidus et celui de la construction d'un modèle empirique sans modèle théorique explicatif.

Etant données que nous cherchons le lien causal entre l'ouverture extérieure, le capital humain et l'emploi, nous avons les variables endogènes suivantes:

- Logarithme d'ouverture extérieure (LOUV): suivant certaines études empiriques (Oliver et Sébastien, 1997 ; Combes, Guillomont, Jeanneney et Motel, 2000), l'ouverture extérieure est mesurée par les flux d'exportation et d'importation de biens et services sur PIB. Tel sera aussi le cas dans notre étude. L'ouverture extérieure s'intéresse aux mouvements des importations et des exportations. Ainsi, sont inclus dans les exportations et importions d'un pays des biens à savoir : les marchandises en générales, les biens importés et exportés pour transformation. Et des services tels que : les transports, les voyages, les services de communication, bâtiment et travaux publics, d'assurance, financiers, d'informatique et d'information, etc. (Ondo Ossa, 1999). En effet, l'ouverture par le commerce et les investissements directs étrangers (IDE), semble être aujourd'hui un catalyseur de la 
croissance économique tant dans les pays émergents que dans les pays développés. A cet effet, nous nous attendons à un signe positif en introduisant cette variable.

Logarithme du capital humain (LKH): il s'agit en effet du logarithme de la population scolarisée du niveau secondaire. Autrement dit-il s’agit du total des inscriptions dans un niveau spécifique d'éducation, sans distinction d’âge, exprimé en pourcentage de la population officiellement scolarisable au même niveau pour une année scolaire donnée. Le capital humain traduit aussi le niveau de qualification de la main d'œuvre et entraine une augmentation de la productivité de celle-ci. Un pays hôte qui dispose d'un stock de capital humain suffisamment qualifié attire nécessairement les flux d'IDE entrants. La littérature empirique nous montre que, un niveau d'éducation élevé renforce la capacité d'un pays à imiter et utiliser les technologies découvertes dans les pays avancés (Benhabib \& Spiegel, 1994). Dans le sens qu'une main d'œuvre éduquée est meilleure dans la création et l'adoption de nouvelles technologies. En conséquence, une effusion des IDE due à la qualification du capital humain peut avoir une conséquence directe sur l'augmentation du niveau de l'emploi dans le pays hôte. Le signe attendu est donc positif.

- Le logarithme du volume d'emploi (LEMP) : selon l'OCDE, c'est la proportion de personnes disposant d'un emploi parmi celles en âge de travailler (15 à 64 ans). Le volume d'emploi reflète la capacité d'une économie à utiliser ses ressources en main-d'œuvre. De nombreux économistes, et par exemple l'OCDE, estiment que l'emploi est une mesure plus pertinente que le taux de chômage pour évaluer la situation du marché du travail d'un pays. La littérature empirique nous montre que l'emploi est fortement influencé par le niveau d'éducation ou de scolarisation des individus et le degré d'ouverture. L'emploi en effet constitue un préalable au développement économique et social de tout pays. Tout comme l'ouverture et le capital humain influenceraient l'emploi, de même, le fait qu'un individu possède déjà un emploi pourrait en retour lui permettre d'accroitre ses potentialités en investissant sur son capital humain dans le but d'être plus compétitif dans un monde toujours en perpétuelle mutation. Par ce fait, le signe attendu est positif.

\section{ii. Variables exogènes}

Le logarithme du taux d'inflation (LINF) : l'inflation correspond à une hausse généralisée du niveau des prix. Elle est mesurée par l'indice des prix à la consommation. Pour calculer l'inflation au sein 
d'un pays ou d'une zone géographique, on calcule la variation en pourcentage de l'indice entre deux périodes données. C’est ainsi que l'on obtient le taux d'inflation mensuel et annuel. L'inflation tire son origine de la création monétaire. Cette création monétaire se mesure avec le niveau de la masse monétaire qui est en perpétuelle augmentation. Mais, une augmentation de la masse monétaire ne signifie pas forcément qu'il y'a inflation. Ce qui conduit à l'inflation, c'est une augmentation plus rapide de la masse monétaire par rapport aux richesses produites (que l'on mesure avec le PIB).L'inflation est choisie comme proxy afin de saisir le niveau de stabilité macroéconomique d'un pays quelconque. L'évolution du taux d'inflation à un impact direct sur l'activité économique d'un pays. Il affecte négativement les transactions avec l'extérieur. Pour cette raison, le signe attendu est négatif.

- Le logarithme des flux d'investissement intérieur brut (LTIB) : cet indicateur regroupe en son sein les investissements directs étrangers, les investissements privés domestiques et les investissements publics. L'investissement est un déterminant important pour tout pays qui souhaiterait relancer son activité de production, accroitre son niveau d'éducation ou de formation du capital humain et améliorer son niveau d'emploi. En 1976, le chancelier Helmith Schmitt remettait à l'honneur la politique de l'offre déclarant devant Bundestag : "les profits d'aujourd'hui font les investissements de demain qui font les emplois d'après-demain.» Cette relation a été vérifiée historiquement. Car, durant les trentes glorieuses, on a observé une corrélation entre le niveau d'investissement, la croissance et l'emploi. Les pays qui affichaient les taux d'investissement les plus élevés présentaient de même des plus hauts taux de croissance et d'emploi. L'investissement est donc une variable incontournable dans un processus de développement. L'augmentation de son taux est une source d'attraction des capitaux extérieurs et d'augmentation d'emplois. Le signe attendu pour cette variable est positif.

Le récapitulatif des signes attendus

\section{3- Les signes attendus des variables}

\begin{tabular}{|c|c|c|c|c|c|}
\hline & $\mathrm{LOUV}_{\mathrm{t}}$ & $\mathrm{LKH}_{\mathrm{t}}$ & LEMP $_{t}$ & LINF $_{t}$ & LTIIB $_{t}$ \\
\hline $\mathrm{LOUV}_{\mathrm{t}}$ & & + & + & - & + \\
\hline $\mathrm{LKH}_{\mathrm{t}}$ & + & & + & & + \\
\hline LEMP $_{t}$ & + & + & & & + \\
\hline $\begin{array}{l}\text { LINF }_{t} \\
\text { LTIIB }_{t}\end{array}$ & $\overline{+}$ & + & + & & \\
\hline
\end{tabular}




\section{Test de racine unitaire}

La spécification d'un modèle nécessite que les variables soient stationnaires, sinon les tests classiques ne seront pas valables et on court le risque d’une régression fallacieuse. La difficulté réside dans le fait que la plupart des données temporelles macroéconomiques sont des réalisations de processus non stationnaires (Nelson \& Plosser, 1982). Toutefois, l'étude de la stationnarité est importante, car elle permet aussi de voir si les moyennes et les variances de séries étudiées sont stables dans le temps. Mais, cela n’est pas toujours le cas, car il arrive souvent dans la réalité que l'on fasse face à des séries non stationnaires. Nous utiliserons dans notre étude le test de Dickey-Fuller Augmented (ADF) pour tester la stationnarité de nos séries. En effet, contrairement aux autres tests (test de Phillips-Perron, test de Kwiatkowski, test de KPSS), si le terme d'erreur est autocorrélé, alors le test d'ADF consistera simplement à augmenter les trois équations de DickeyFuller en y ajoutant des valeurs décalées de la variable dépendante. Il consiste pour une p-value calculée et inférieur au niveau de signification alpha, à rejeter l'hypothèse nulle (présence d'une racine unitaire : la série est non stationnaire) et à accepter l'hypothèse alternative (absence d'une racine unitaire : la série est stationnaire). Après la mise en œuvre du test d'ADF, il est nécessaire de déterminer le retard optimal du polynôme autorégressif.

Détermination du nombre de retards optimaux

Pour effectuer notre estimation, nous avons besoin de déterminer le nombre de retard à introduire. Le choix du nombre maximal de retards susceptibles d'apporter de l'information à l'estimation du VAR se fait à partir de l'interprétation des différents critères d'information. Le retard maximal étant celui qui minimise les critères d'information d'Akaike (AIC), Hannan-Quinn (HQ) ou de Schwartz (SIC).

\section{4-TESTS DE NON CAUSALITE DE TODA ET YAMAMOTO}

Pour la présente étude, l'analyse de la direction de la causalité entre l'ouverture, le capital humain et l'emploi nous amène à considérer plusieurs concepts : d'abord le concept de causalité au sens de Granger qui stipule qu'une variable cause une autre si et seulement si les valeurs présentes et passées de la dernière permettent de mieux prédire les valeurs de la première. Pour ce faire, il convient de commencer par tester la stationnarité des variables tels que nous l'avons mentionné et présenté plus haut. Après avoir testé la stationnarité des différentes variables, la plupart des études nous amènent ensuite à tester la Co intégration entre les différentes variables. L'objectif de ce second test est de chercher l'existence d'une relation de long terme entre les variables. Ensuite, il faudra estimer un modèle à correction d'erreurs. 
Mais, après l'analyse de la conception de Toda et Yamamoto (1995), le fait de passer par ces différentes étapes accroit les risques de biais dans l'étude. En effet, ils montrent que cette analyse séquentielle de la causalité peut engendrer une accumulation de petits biais qui vont compromettre la fidélité des résultats obtenus. Tout d'abord, la petite taille des échantillons face à la puissance des tests de racine unitaire et ensuite, la sensibilité des tests de cointégration aux choix d'un certain nombre de paramètres de nuisance face toujours à un échantillonnage réduit. Selon toujours Toda et Yamamoto, l'objectif de l'économiste n'est pas de savoir si les variables sont intégrées ou cointégrées mais plutôt de tester la soutenabilité ou la viabilité d'hypothèses théoriques. Autrement dit, Le test de causalité développé par Toda et Yamamoto permet d'étudier la causalité dans un système de variables non stationnaires intégrées d'ordres différents. La nécessité d'étudier la cointégration est outre passée par une surparamétrisation non optimale du VAR. L'unique condition reste toutefois que l'ordre du VAR soit supérieur à l'ordre maximal d'intégration possible des variables.

Ainsi, s'inspirant des analyses de Toda et Yamamoto, nous procéderons à une analyse non séquentielle des tests de causalité. Autrement dit, au test de non causalité de Toda et Yamamoto. Ce test se réalisera en deux étapes : tout d'abord, il s'agira de déterminer le nombre de retards optimal $(k)$ du processus VAR en niveau, cela se fera grâce aux critères d'information. Cette procédure de correction du VAR en niveau tient compte d'une éventuelle relation de cointégration sans pour autant l'étudier expressément, permettant ainsi de valider l'existence d'une causalité en un unique test. Ensuite, il faudra estimer le modèle VAR en niveau d'ordre $\mathrm{p}=\mathrm{K}+\mathrm{dmax}$ avec dmax l'ordre d'intégration maximale des séries.

En somme, il était question pour nous dans cette partie, d'élaborer la démarche méthodologique adéquate nous permettant d'atteindre l'objectif de notre étude. Partant de la théorie économique néoclassique qui établit la relation entre la production, l'emploi et le capital, nous avons élaboré notre modèle économétrique à estimer. Toutefois, pour mieux analyser la causalité entre nos variables, la modélisation VAR est celle que nous avons trouvée mieux adaptée. En outre, pour tester la stationnarité des séries, nous avons choisi le test de Dickey-Fuller (ADF). Enfin, pour tester la causalité, le test de non causalité Toda et Yamamoto est celui que nous retenons. Dans la prochainepartie, il sera question pour nous de procéder aux analyses sur les variables de notre modèle et de donner une interprétation.

Dans le cas du crédit scoring c'est le processus d'assignation d'un score (note) à un emprunteur potentiel pour estimer la performance future de son prêt (Flaman, 1997). Il est défini selon R. Rayon A. (2007) comme étant le recours aux modèles statistiques en vue de transformer des données 
(qualitatives et quantitatives) en indicateurs numériquesmesurables à des fins d'aide à la décision d'octroi ou de rejet de crédit. C'est un outil d'aide à la prise de décision d'octroi de crédit des établissements financiers.

Pour la suite de l'étude, nous insisterons sur la méthode du diagnostic financier de Dupont et son application à MIGEG S.A. dans le système de Dupont, le Ratio central servant à mesurer la performance de l'entreprise s'appelle le rendement de l'avoir. Il se calcule comme suit :

\section{Équation 2:Rendement de l'avoir $=\frac{\text { Bểnêfice net }}{A \text { wo: }}$}

Le ratio nous donne la mesure du bénéfice retiré des activités de l'entreprise pour rapport à la mise de fonds comptables des propriétaires (qui est représentée ici par l'avoir : essentiellement, ce qu'ils ont investi dans l'entreprise au départ plus tous les bénéfices réalisés au fil des ans et qu'ils y ont réinvestis).

Si le choix du bénéfice net au numérateur va de soi, celui de l'avoir au dénominateur demande de préciser le point de vue adopté dans l'analyse. Ici, le choix de l'avoir signifie que le point de vue adopté est celui du propriétaire.

Considéré comme le point de départ le point de vue des propriétaires a beaucoup de sens pour un gestionnaire dont l'objectif est la maximisation de leur richesse et c'est l'optique du gestionnaire que nous adopterons ici. Cependant, le modèle proposé ici est très complet et permettra d'adopter également d'autres points de vue comme celui de l'ensemble des fournisseurs de capitaux.

Bien qu'il s'agisse d'un seul ratio, le rendement de l'avoir permet, lorsqu'il est calculé sur plusieurs années, de dégager certaines tendances.

A titre d'illustration considérons ici les valeurs prises sur une période de 10 années par le ratio de remboursement de l'avoir de deux agences MIGEG S.A très différents qu'on nomme Agence A et Agence B.

Tableau 1. Agence MIGEG S.A A

\begin{tabular}{|l|l|l|l|l|l|l|l|l|l|l|}
\hline Années & 2005 & 2006 & 2007 & 2008 & 2009 & 2010 & 2011 & 2012 & 2013 & 2014 \\
\hline Bénéfice & 8 & 8 & 6 & 9 & 9 & 8 & 10 & 11 & 10 & 11 \\
\hline Avoir & 55 & 57 & 49 & 62 & 70 & 68 & 59 & 70 & 73 & 69 \\
\hline Rendement & 0.14 & 0.14 & 0.12 & 0.15 & 0.13 & 0.12 & 0.17 & 0.16 & 0.14 & 0.16 \\
\hline
\end{tabular}

Source: documentation MIGEG S.A.A 2011 
Figure 1. Performance d'Agence A

Graphique de la performance d'Agence MIGEG S.A A suivant tableau ci-dessus

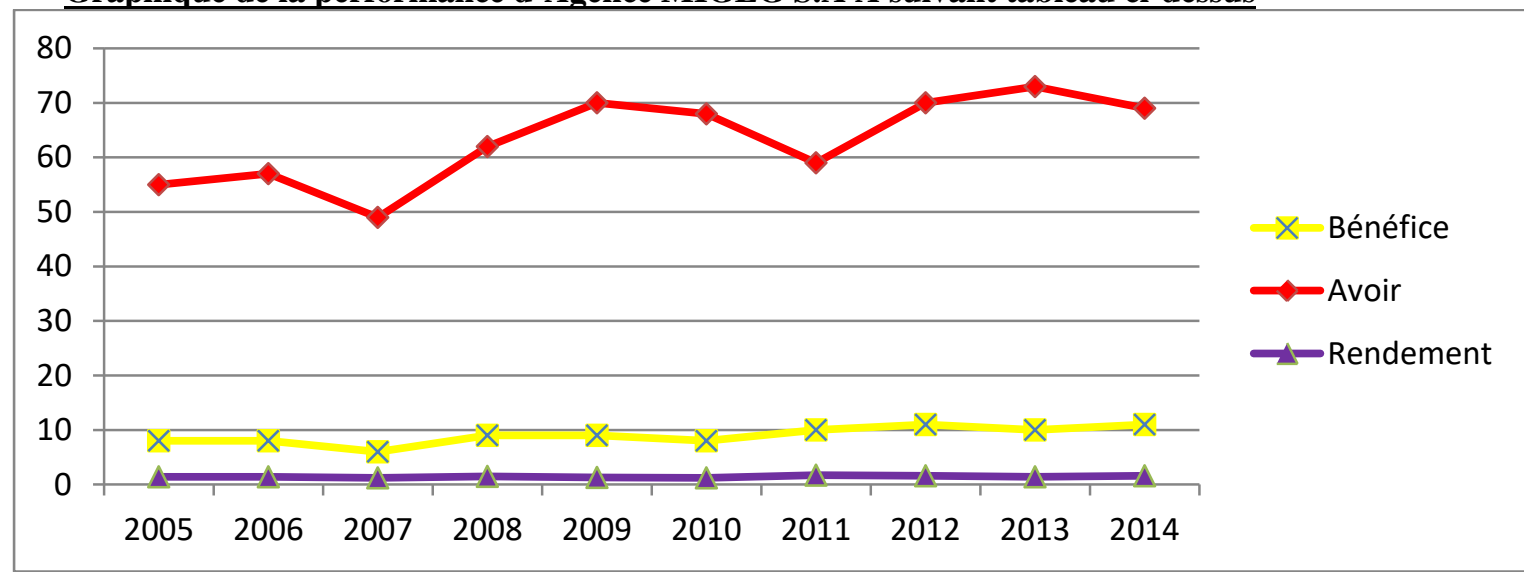

Sources: Auteur 2011

Tableau 4. Agence MIGEG S.A B

\begin{tabular}{|l|l|l|l|l|l|l|l|l|l|l|}
\hline Années & 2005 & 2006 & 2007 & 2008 & 2009 & 2010 & 2011 & 2012 & 2013 & 2014 \\
\hline Bénéfice & 14 & 8 & 10 & 12 & 15 & 16 & 17 & 19 & 19 & 19 \\
\hline Avoir & 70 & 65 & 65 & 69 & 70 & 70 & 75 & 79 & 80 & 80 \\
\hline Rendement & 0.20 & 0.12 & 0.15 & 0.17 & 0.21 & 0.22 & 0.22 & 0.24 & 0.22 & 0.22 \\
\hline
\end{tabular}

Source : MIGEG S.A data en millions de FCFA 2012

Figure 2. Performance d'Agence MIGEG S.A B

Graphique de la performance d'Agence MIGEG S.A B suivant tableau ci-dessus

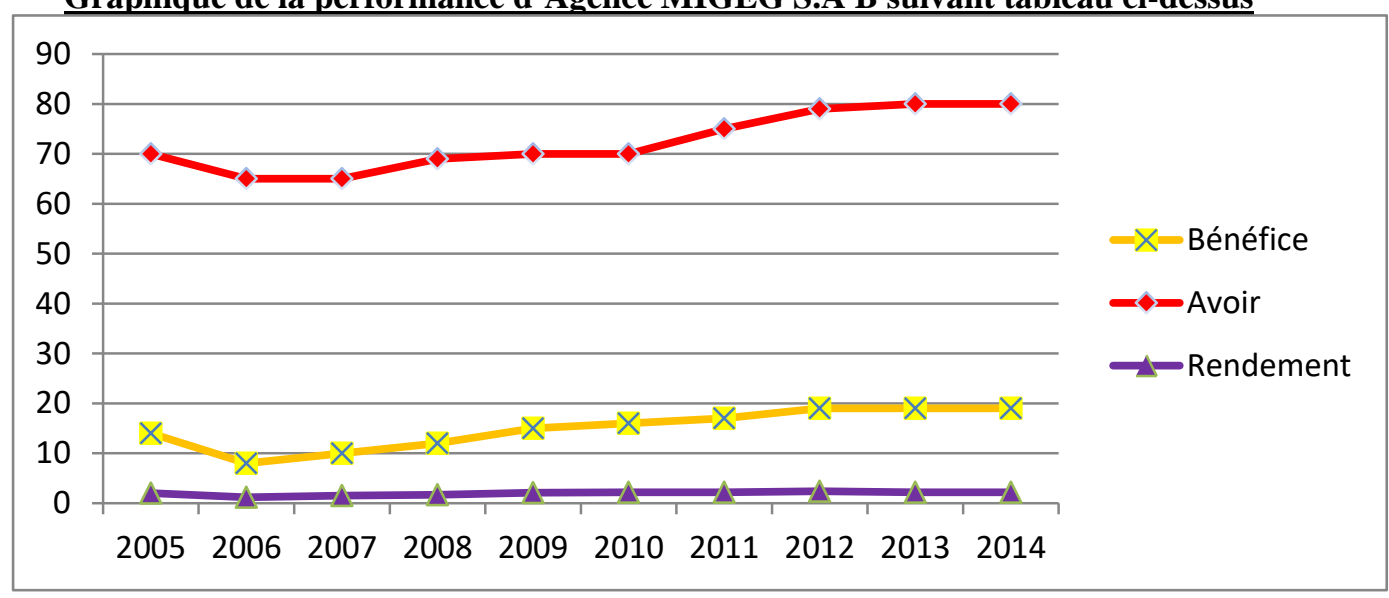

Sources : Auteur 2012

On constate que le rendement de l'avoir de l'agence B est plus élevé que celui de l'agence A pour certaines années alors qu'il est plus faible pour d'autres. Par ailleurs, il est plus stable à travers la période considérée. Il s’agit bien sûre d'une information partielle mais tout de même révélatrice 
entre autre sur la variabilité de la performance financière de chacune des agences à travers les 10 années.

D- Le niveau de décomposition

1. L’équation standard : les 3 premiers ratios et leur signification.

La décomposition de premier niveau du ratio central donne l’équation suivante

$$
\frac{\text { Bènèfice net }}{\text { Avoir }}=\frac{\text { Bènéfice net }}{\text { Ventes }} * \frac{\text { Ventes }}{\text { Actifs }} * \frac{\text { Actifs }}{\text { Avoir }}
$$

Rendement de l'avoir
(1) Marge nette
(2)

Rotation des actifs Levier financier

Le premier ratio, que l'on appelle ratio marge nette, constitue le premier point d'intérêt du système de Dupont, le premier facteur explicatif du rendement. Il renseigne sur la rentabilité des ventes de l'entreprise, c'està-dire sur la quantité de bénéfice net que génère chaque franc de vente. Idéalement, l'entreprise souhaitera que ce ratio soit le plus élevé possible puisque cela signifie qu'elle retire le maximum de bénéficesdel'effort de vente.

Équation 3: Marge nette $=\frac{B \text { ênêfice net }}{\text { Ventes }}$

On peut faire ressortir ici les éléments qui influenceront la valeur de ce ratio. Tous les éléments qui composent l’état des résultats sont en cause : les ventes (c'est donc la quantité vendue et les prix de vente) et les diverses charges encourues pour réaliser ces ventes. Parmi les champs, on retrouverales coûts (d'achat ou de fabrication) des marchandises vendues, les frais de vente et l'administration, l'amortissement des immobilisations de l'entreprise de même que les intérêts en commun sur la dette que l'entreprise a contracté et les impôts chargés à l'exercice.

L’objectif du gestionnaire dans cette première étape de l'analyse, sera de déterminer si la valeur prise par le ratio de marge nette est suffisamment élevée, d’identifier les véritables cours de sa baisse ou de sa hausse et, dans une perspective de gestion de connaitre les variables sur lesquelles il peut exercer un certain contrôle de la même façon que les actions à prendre afin d'obtenir la margenette la plus grande possible.

2. Le ratio de notation des actifs

Il constitue le deuxième facteur explicatif du rendement de l'avoir du système de Dupont. Et sert à mesurer l'efficacité de la gestion des actifs et représente la quantité de dollars de vente que peut générer chaque dollar 
investi dans les actifs del'entreprise. Ce ratio fait donc un lien entre les deux états financiers principaux, à savoir le bilan et l'état des résultats. Idéalement, on voudrait encore que ce ratio soit le plus élevé possible pour qu'il signifie alors que les actifs sont utilisés au maximum, ce qui, couplé à la marge la plus élevée possible aura un impact à la hausse sur le rendement de l'avoir qu'on note :

\section{Équation 4: Rotation des actifs $=\frac{\text { Ventes }}{\text { Actifs }}$}

Soulignons dès à présent un phénomène de compensation qui existe entre ces deux premiers ratios.

3. Le ratio du levier financier

Il renseigne quant à lui sur la proportion des actifs de l'entreprise qui est financée par ses propriétaires. Pour mieux en comprendre le sens, il suffit de remplacer le numérateur par son équivalent en termes de source fonds, soit dette + avoir de sorte que le ratio devient:

\section{Équation 5: Le Ratio du Levier Financier}

\section{Dette + avoir}

\section{Avoir}

Plus ce ratio sera élevé, plus le recours à l'effet de levier est grand plus le faux de remboursement de l'avoir sera grand, toute chose étant égale par ailleurs.

\section{Figure 3. Décomposition du Système de Dupont}

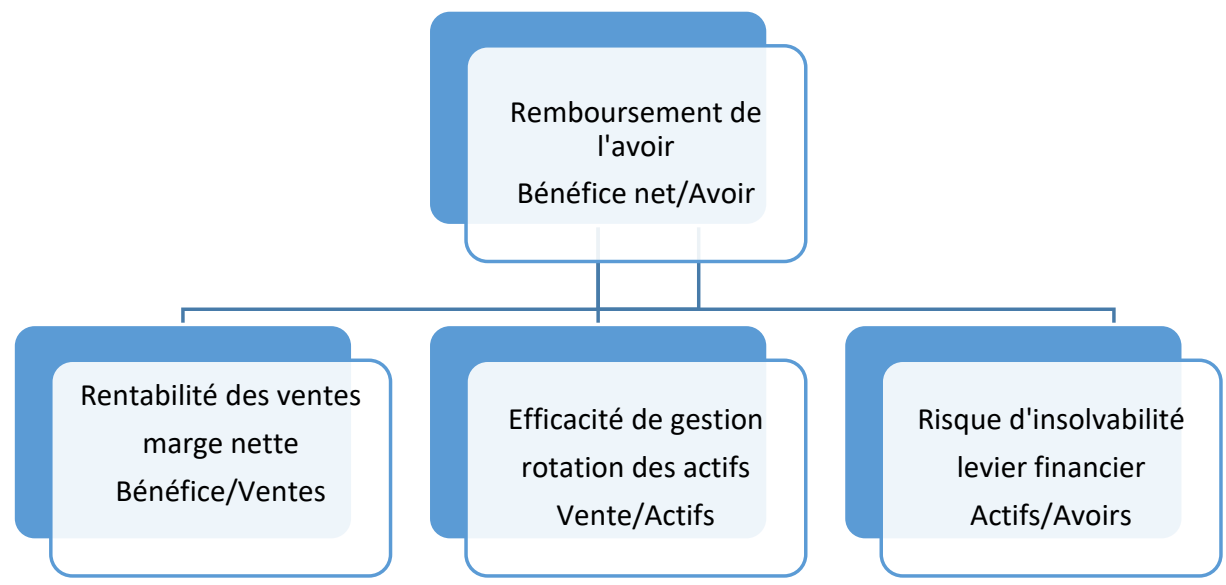

Sources : Auteur 2013 


\section{II-CONSEQUENCE DU MODELE SUR LE CREDIT A RISQUE}

En entreprise : Le cas de MIGEG S.A.

A- Situation avant implémentation du modèle.

Le tableau ci-dessous récapitule l'activité de crédit de la MIGEG avec le PME durant les six derniers mois avant l'implémentation du modèle.

Tableau 5. Récapitulatif des crédits avant implémentation du modèle (6 mois)

\begin{tabular}{|l|l|l|l|l|}
\hline Mois & Echéance & Crédits demandés & Crédits octroyés & Montant en FCFA \\
\hline Juillet & 3 mois & 25 & 23 & 10.537 .320 \\
\hline Août & 3 mois & 29 & 24 & 80.382 .301 \\
\hline Septembre & 3 mois & 19 & 18 & 100.654 .396 \\
\hline Octobre & 3 mois & 34 & 30 & 101.125 .764 \\
\hline Novembre & 3 mois & 63 & 57 & 40.015 .000 \\
\hline Décembre & 3 mois & 77 & 66 & 30.280 .214 \\
\hline Totaux & 3 mois & 247 & 218 & 362.995 .095 \\
\hline
\end{tabular}

Source: rapport trimestrielle de l'activité de crédit de la MIGEG ( $3^{\text {eme }}$ es trimestre 2015)

Figure 4. Crédits avant implémentation du modèle (Graphique 1a)

Graphique 1a. Récapitulatif des crédits avant implémentation du modèle pour les Crédit octroyé contre Crédit demandé par Echéances de 3 mois suivant table3

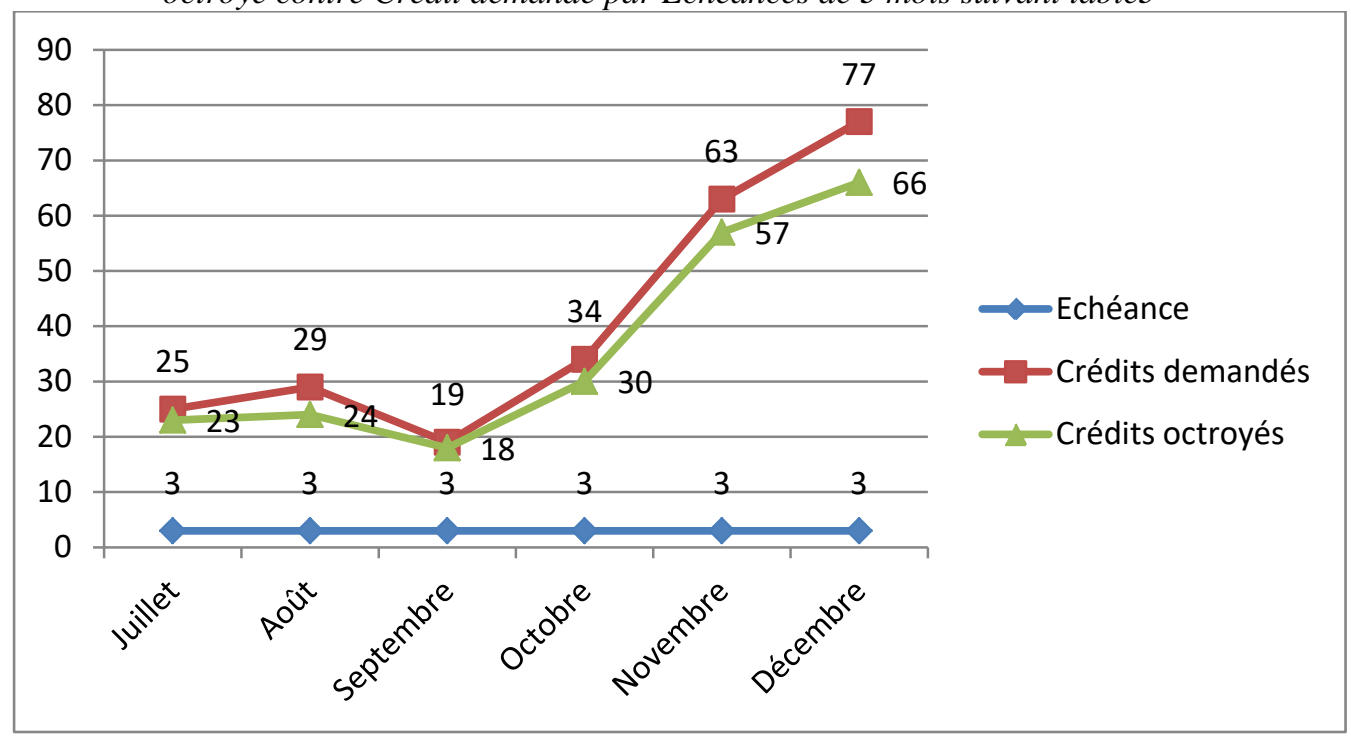

Source: auteur 2012 
Figure 5. Credits avant implementation du modele (Graphique 1b)

Graphique 1b:Récapitulatif des crédits avant implémentation du modèle pour les Montant en F Xaf suivant table 3

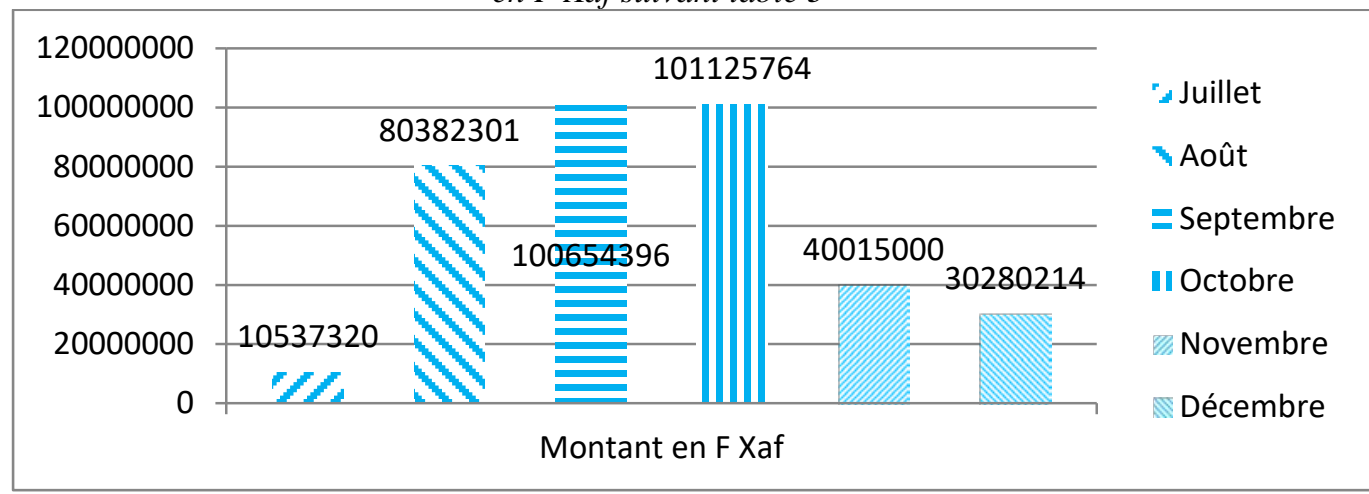

Sources: Auteur 2012

Tableau 6. Etats des remboursements avant implémentation du modèle

\begin{tabular}{|l|l|l|l|}
\hline Mois & Montant accordé & Montant remboursé & Montant non remboursé \\
\hline Juillet & 10.537 .320 & 9.537 .320 & 1.000 .000 \\
\hline Août & 80.382 .301 & 75.688 .953 & 4.693 .348 \\
\hline Septembre & 100.654 .396 & 93.974 .964 & 6.679 .432 \\
\hline Octobre & 101.125 .764 & 100.000 .909 & 1.124 .855 \\
\hline Novembre & 40.015 .000 & 35.047 .990 & 4.967 .010 \\
\hline Décembre & 30.280 .214 & 28.037 .344 & 2.242 .870 \\
\hline Totaux & 362.995 .095 & 342.287 .480 & 20.707 .615 \\
\hline
\end{tabular}

Source: Rapport trimestriel de la MIGEG décembre 2015.

Figure 6. Etats des remboursements avant implémentation du modèle (Graphique 2a)

: Graphique 2a. états des remboursements avant implémentation du modèle pour les Montant accordé contre les Montant rembourséet non remboursé suivant table4 


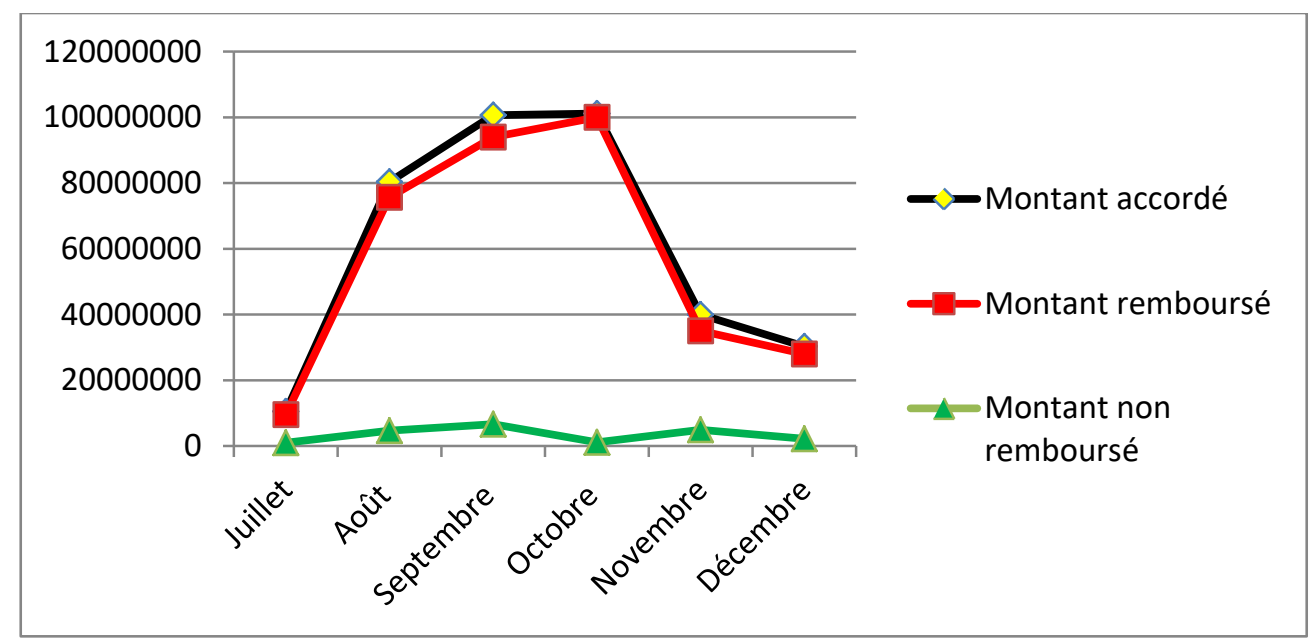

Figure 7. Etats des remboursements avant implémentation du modèle (Graphique 2b)

Sources: Auteur 2012

Graphique 2b. états des totaux de remboursements avant implémentation du modèleles Montant accordé contre les Montant remboursé et non remboursé suivant table4

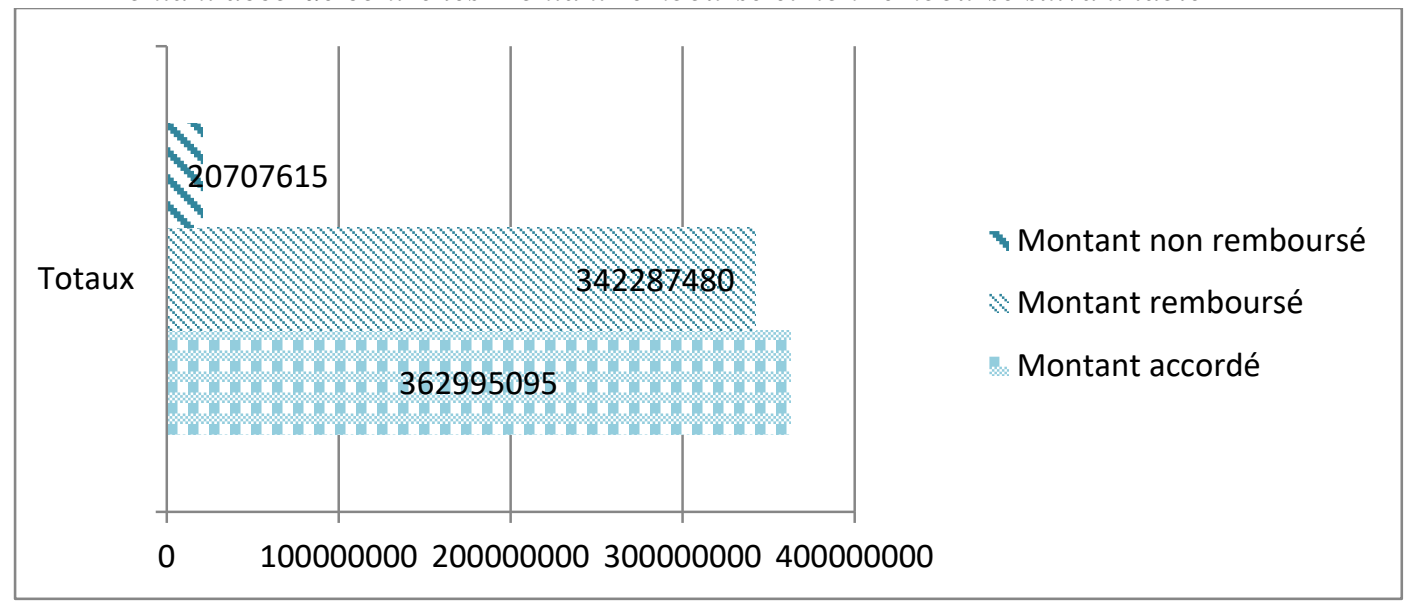

Sources: Auteur 2012

Le constat qui se dégage durant les six mois est que l'EMF est très réticente quant à accorder les crédits aux clients. Sur 184 demandes de crédit, 56 seulement ont été accordées. Soit un taux de 30,43\% et pour un montant de 253.149.406 FCFA.

Le pourcentage de crédit remboursé est de 78,86\%, soit 199.654 .718 FCFA sur les 253 millions empruntés.

Le pourcentage de crédit non remboursé est de 24,17\%.

Ce pourcentage de crédit non remboursé est très important, ce qui rend l'EMF réticente quant à accorder encore plus de prêts. Cela entraine une baisse de confiance pour les autres prêts et un manque à gagner pour 
l'entreprise du moment où nous savons que l'activité de crédit est l'activité la plus rentable de L'EMF.

B- La situation après implémentation du modèle.

Les crédits octroyés ont été évalués selon le processus de la méthode du diagnostic amélioré et de Dupont.

Tableau 7. Récapitulatif des créditsaprèsimplémentation du modèle

\begin{tabular}{|l|l|l|l|l|}
\hline Mois & Echéances & Crédit demandé & Crédit octroyé & Montant en FCFA \\
\hline Juillet & 3 mois & 25 & 23 & $\mathbf{1 0 5 3 7 3 2 0}$ \\
\hline Août & 3 mois & 29 & 24 & $\mathbf{8 0 3 8 2 3 0 1}$ \\
\hline Septembre & 3 mois & 19 & 18 & $\mathbf{1 0 0 6 5 4 3 9 6}$ \\
\hline Octobre & 3 mois & 34 & 30 & $\mathbf{1 0 1 1 2 5 7 6 4}$ \\
\hline Novembre & 3 mois & 63 & 57 & $\mathbf{4 0 0 1 5 0 0 0}$ \\
\hline Décembre & 3 mois & 77 & 66 & $\mathbf{3 0 2 8 0 2 1 4}$ \\
\hline Totaux & & 247 & 218 & $\mathbf{3 6 2 . 9 9 5 . 0 9 5}$ \\
\hline
\end{tabular}

Source : Rapport trimestrielle de l'activité de crédit de la MIGEG ( ${ }^{\mathrm{e}}$ et $4^{\mathrm{e}}$ trimestre 2015).

Figure 8. Créditsaprèsimplémentation du modèle (Graphique 3a)

Graphique 3a. Récapitulatif des crédits après implémentation du modèle pour les Crédit octroyé contre Crédit demandé par Echéances de 3 mois suivant table5

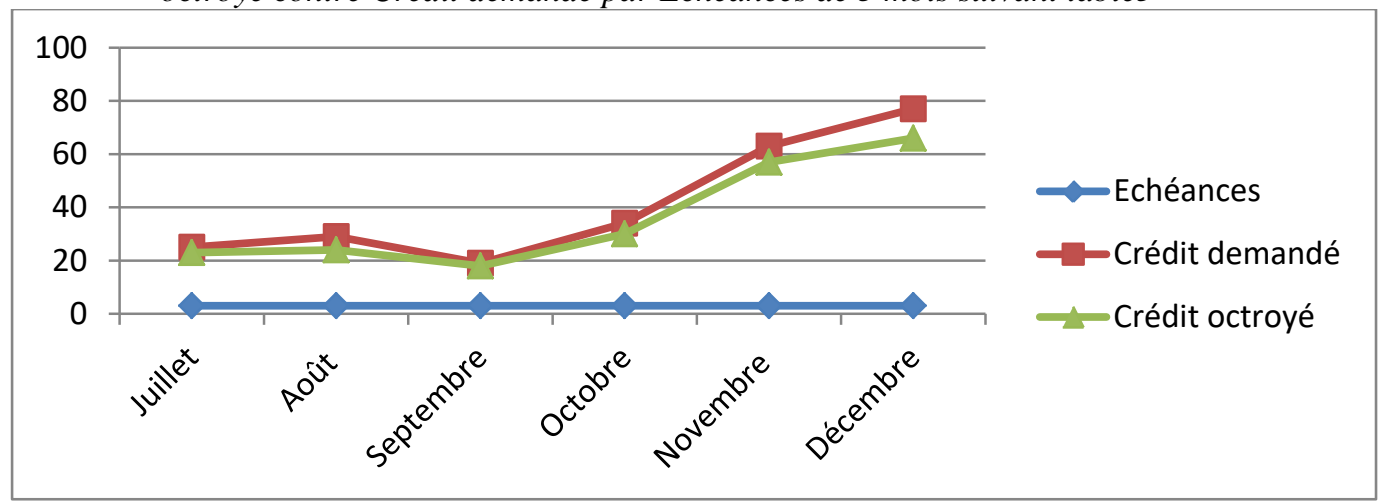

Sources: Auteur 2013

Figure 9. Créditsaprèsimplémentation du modèle (Graphique 3b)

Graphique 3a. Récapitulatif des crédits après implémentation du modèle pour les montant total Crédit octroyé contre Crédit demandé par Echéances de 3 mois suivant table 5 


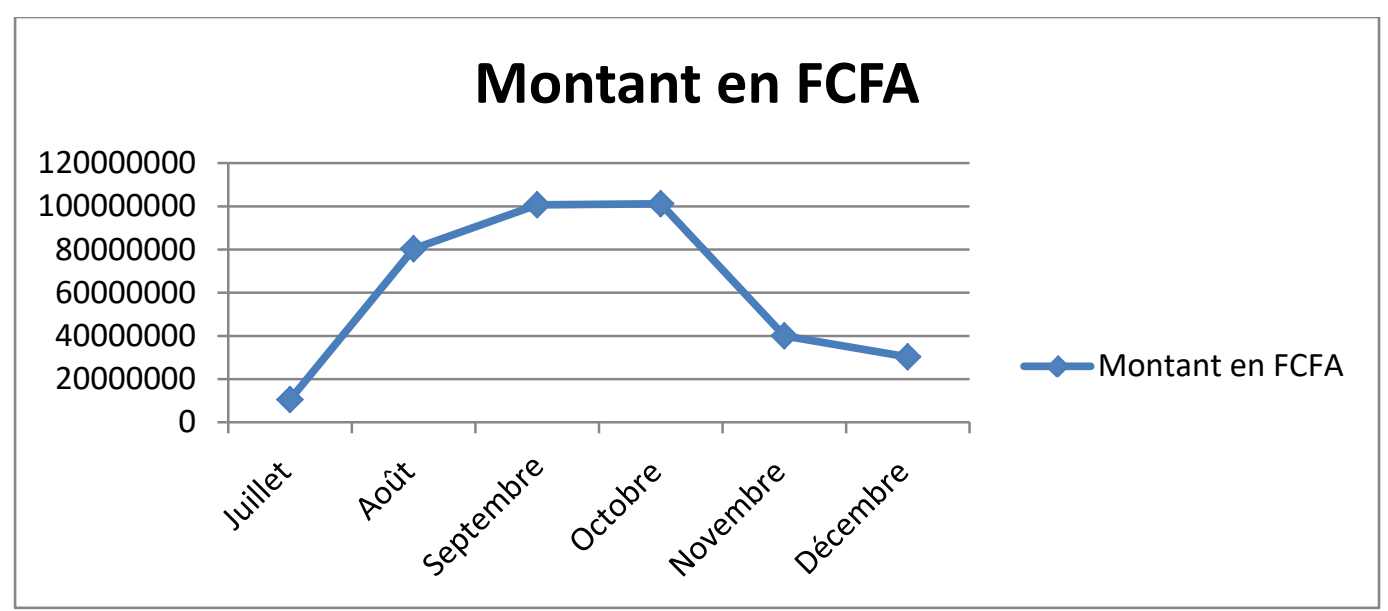

Sources: Auteur 2012

Tableau 8. Etats des remboursements après implémentation du modèle

\begin{tabular}{|l|l|l|l|l|}
\hline Mois & Echéances & Crédit demandé & Crédit octroyé & Montant en FCFA \\
\hline Juillet & 3 mois & 25 & 23 & $\mathbf{1 0 5 3 7 3 2 0}$ \\
\hline Août & 3 mois & 29 & 24 & $\mathbf{8 0 3 8 2 3 0 1}$ \\
\hline Septembre & 3 mois & 19 & 18 & $\mathbf{1 0 0 6 5 4 3 9 6}$ \\
\hline Octobre & 3 mois & 34 & 30 & $\mathbf{1 0 1 1 2 5 7 6 4}$ \\
\hline Novembre & 3 mois & 63 & 57 & $\mathbf{4 0 0 1 5 0 0 0}$ \\
\hline Décembre & 3 mois & 77 & 66 & $\mathbf{3 0 2 8 0 2 1 4}$ \\
\hline Totaux & & 247 & 218 & $\mathbf{3 6 2 . 9 9 5 . 0 9 5}$ \\
\hline
\end{tabular}

Source : Rapport trimestriel de la MIGEG de décembre 2015.

Figure 10. Les remboursements après implémentation du modèle (Graphique 4a)

Graphique 4a. Etat de remboursement après implémentation du modèle pour les Crédit octroyé contre Crédit demandé par Echéances de 3 moisdu table6 


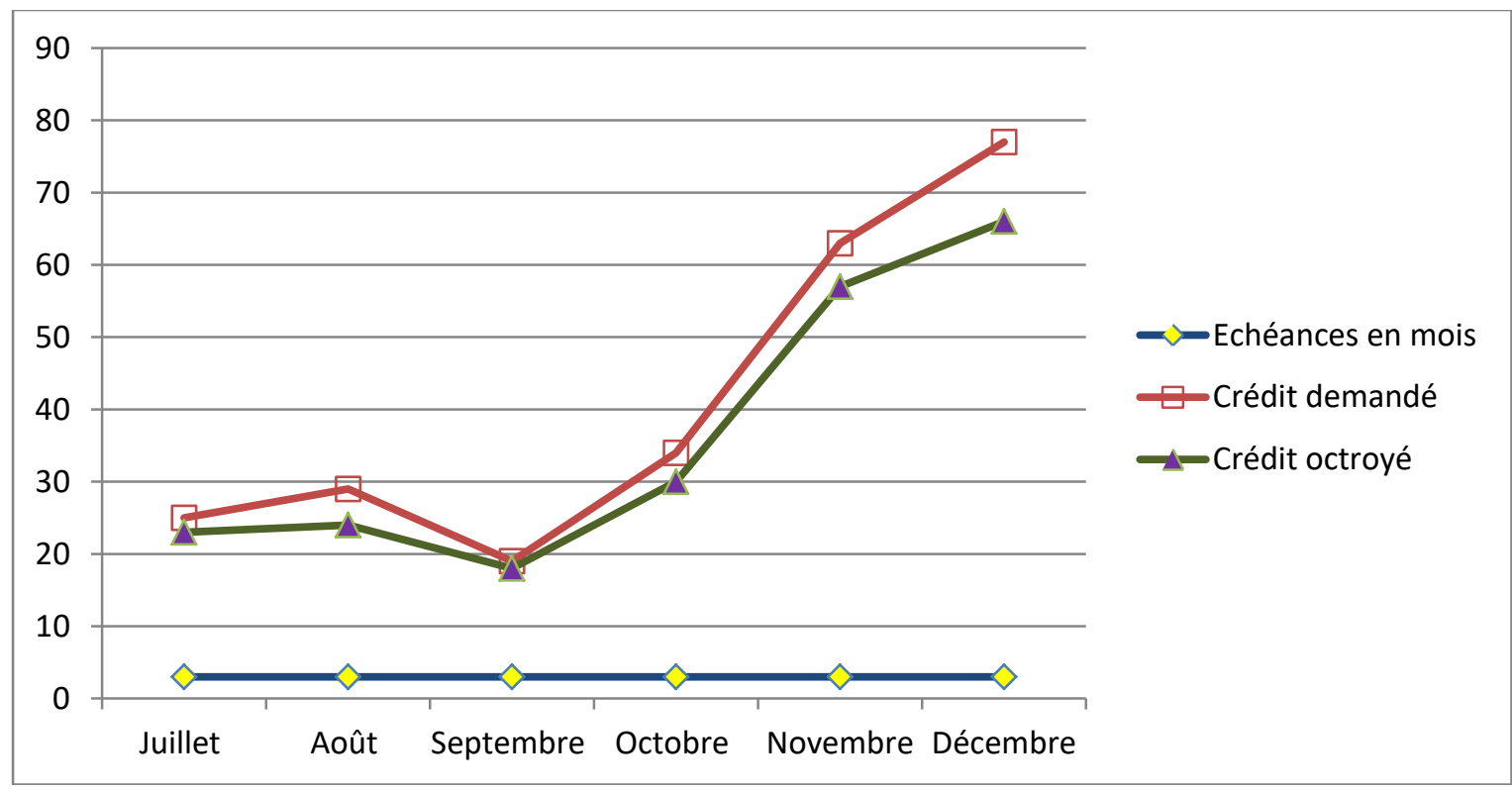

Sources: Auteur 2012

Figure 11. Les remboursements après implémentation du modèle (Graphique 4b)

Graphique 4a. Etat de remboursement après implémentation du modèle pour les Montant

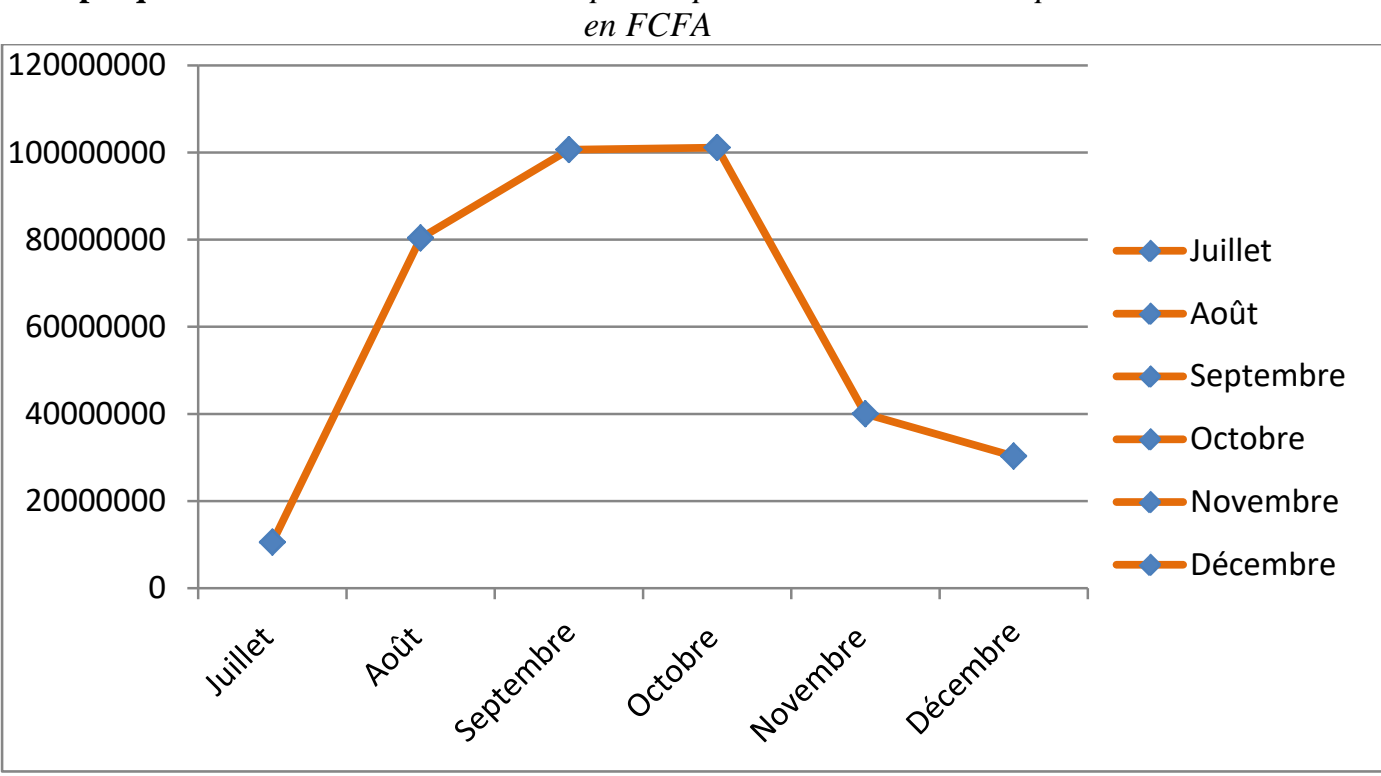

Sources: Auteur 2012

Des deux derniers tableaux ci-dessus, on remarque qu'avec la mise en place du modèle, l'EMF est plus apte à accorder du crédit, ce qui se manifeste à travers les résultats obtenus:

- 247 demandes, 218 accordées, soit un taux de 88,25\% 
- Les montants des crédits déjà remboursés à l'échéance de 6 mois est 342.287.450 FCFA soit un taux de 94,29\%

- Le montant des créances en souffrance est nettement en domination et porte un montant de 2077615 FCFA, soit un taux de remboursement de 5,70\%

\section{CONCLUSION GENERALE}

En partant des objectifs qui insistent sur le levier financier pour préfinancer les risques et les ventes élevées, devant assurer une trésorerie viable, le modèle mettant en relation l'ouverture extérieur, l'emploi, le capital humain, l'inflation et l'investissement intérieur; ou même les résultats centrés sur l'ouverture extérieur, l'emploi, l'investissement intérieur devant être soutenu par les ventes élevées et le préfinancement des risques d'ouverture extérieur et les investissements générateur d'emplois, fait du modèle proposé, un modèle efficace mais ne pouvant se juger complet. Pour le rendre plus efficace, il y a quelques recommandations concernant certains cas que nous nous proposons de présenter afin de permettre au gestionnaire de crédit d'être au top de sa quête de minimisation des risques de crédit qu'il accorde, mais aussi aux autorités financières nationales.

-L'intention de l'emprunteur étant difficile à cerner, le chargé de crédit plus qu'un analyste, doit se comporter en véritable psychologue. Il doit être capable d'interpréter les gestes et intentions de l'emprunteur.

-Le gestionnaire doit être proactif afin d'anticiper sur les éventuels incidents financiers de la PME/PMI. Ceci, à travers le préfinancement des risques et surtout en cas d'ouverture extérieur et d'investissements générateur d'emplois.

-De même, l'EMF doit promouvoir des relations constantes et de long terme avec ses clients nouveaux comme anciens, ceci pouvant s'avérer un véritable outil de mesure de la moralité du client.

-Le chargé de crédit doit rester vigilant. La procédure étant longue et très détaillé, il ne doit négliger aucune étape, il doit garder à l'esprit que le risque peut se trouver dans le moindre petit détail et à tous les niveaux du processus.

-Le charger de crédit ne doit pas lessiver l'affinité due à la longue relation qui influence sa décision envers un client. Ce sentiment peut être de nature à baisser les résultats et à favoriser l'octroi des mauvais crédits.

Cette étude mettant en exergue les relations entre les établissements financiers et les PME/PMI, regorge quelques recommandations que nous pouvons donner concernant ces derniers:

-Les PME/PMI ne doivent transmettre aux banquiers que les demandes qui respectent les canaux d'où la nécessité de consultation d'un 
spécialiste, ceci permettrait de faciliter l'analyse du dossier et d'éviter des pertes de temps. De même, les promoteurs se doivent d'être sincères et de bonne foi lors du montage de leur demande de crédit.

-On peut donc dire que la banque des PME mise sur pied par l'Etat du Cameroun va surement contribuer au développement du microcrédit qui est un secteur important pour la croissance de l'économie nationale.

\section{References:}

1. Ambar M. (2007). La gestion du risque de crédit par la méthode RAROC, éd. école Supérieure de Banque, Alger.

2. Anne J. (2000). "Le rationnement du crédit dans les pays en développement, le cas du Cameroun", éd harmattan, Paris.

3. Alioune S. (2000). La compétitivité future des économistes africains. Acte de forum de Dakar, carthala, Amazon.

4. Antoine G. (2003). Economie bancaire, édition publibook, Amazon

5. Anne J. (2000). Le rationnement du crédit dans les pays en developpement, le cas du cameroun, edition harmattan, paris.

6. Ambar M (2007). La gestion du risque de micro crédit,edition, hamattan, paris.

7. Bouyakou F. (2006). " l'entreprise est le financement bancaire », éd harmattan, Paris.

8. Bouyakou F. et al. (2012). « micro finance et réduction de la pauvreté de la femme rurale », ed harmattan, Paris.

9. Bouyop K. et al. (2012). « Micro finance et réduction de la pauvreté de la femme rurale », Harmattan, p.43.

10. Bate (2006). Le guide de la micro finance, edition CLE, yaoundé.

11. Barro \& Sala-i-martin (1995). Public finance in models of economic growth, NBER program, journal of economic growth.

12. Coussergues S. (2002). "Gestion de la banque”, éd Dunod, Paris, P.159.

13. COBAC (2002). règlement CEMAC relatif aux onditions d'exercice et de contrôle de l'activité de la microfinance, éd COBAC, Yaoundé.

14. Diatkure S. (2003). "Les fondements de la théorie bancaire: des textes classiques aux débats contemporains" éd Dunod, Paris, P.39.

15. Fall M. (2011). Mutations et crises de la microfinance, Université Gaston Berger, Saint Louis, Sénégal.

16. Jacques F. (1983). Opérations de banque, Dalloz, Toulouse, p.187.

17. Julien \& Pallaque (1995). La gestion du risque de microcrédit, Edition Dalloz, Toulouse.

18. Lanha (2006). Evaluation du risque de microcrédit, Edition cle, Yaoundé. 
19. Lucas R.E. (1988). On the mechanics of economic development, journal of monetary economics 22, 3-42.

20. Muhammad Y. \& Jolis, Alan (1997). Vers un monde sans pauvreté, Bangladesh, Ed. Jean-Claude Lattès.

21. Pony L. (2012). “Institution financière et réduction de la pauvreté ...”, thèse de Doctorat Phd publiée aux éditions P.A.F en Allemagne.

22. Veyrenc A. (1998). Banque,Bourse et Assurance, édition harmattan, paris.

23. Vernimmen P. (2011). Finance d'entreprise, Edition Dalloz, Toulouse.

24. Williams P. (1958). Microfinance management of risk, journal of political economy, 94(5), 1002-1037. 